\title{
Cerebellar Purkinje cell vulnerability to prenatal nicotine exposure in sudden unexplained perinatal death
}

\author{
Anna M. Lavezzi, Melissa F. Corna, Maria L Repetti, Luigi Matturri \\ "Lino Rossi" Research Centre for the Study and Prevention of Unexpected Perinatal Death and SIDS, Department of Biomedical, \\ Surgical and Dental Sciences, University of Milan, Italy
}

\begin{abstract}
The present study was aimed at supplementing our previous investigations on the morphological features of the Purkinje cells during the autonomic nervous system development, particularly in victims of sudden perinatal death (Sudden Intrauterine Unexplained Death Syndrome and Sudden Infant Death Syndrome), given their crucial role in determining connectivity patterns in the brain as well as in the control of autonomic functions. We highlighted in these pathologies, and precisely in 21 cases of sudden foetal death and 26 cases of sudden infant death, a high percentage of developmental defects of the Purkinje cells such as heterotopia, hypoplasia, hyperplasia, mitotic and/ or shrunken features and abnormal neuronal nuclear antigen expression. These alterations can be interpreted as a result of a defective maturation and/or migration of Purkinje cells in foetal cerebellum, likely consequence of exposure to injuries, particularly to maternal cigarette smoke. Interestingly, we observed in sudden perinatal deaths an association with similar developmental defects of both the dentate and the inferior olivary nuclei. This suggests the existence of a Purkinje-Olivo-Dentate network playing a fundamental role in triggering a sudden death mechanism in perinatal life in the presence of specific risk factors.
\end{abstract}

Key words: Purkinje cells, cerebellar cortex, SIUDS, SIDS, dentate nucleus, inferior olivary nucleus, cigarette smoke.

\section{Introduction}

The human cerebellar cortex is known to undergo rapid changes during the foetal and early postnatal development, changing in thickness as well as undergoing reorganization of its cortical layers [1,14,32].

The timing of these changes has been the subject of our previous investigations [24-26]. In these studies we highlighted in particular, besides the dynamic sequence of the developmental steps, maturational disorders of the cerebellar cortex in sudden intrauter- ine unexplained death and sudden infant death syndromes (SIUDS and SIDS, respectively).

The purpose of this paper was to provide additional information about the Purkinje cells (PCs), given their crucial role in determining connectivity patterns in the brain and, in general, in the coordination of the autonomic functions [31,46].

The PCs in the mature cerebellar cortex are easy to analyse as they are among the largest brain neurons, placed in an ordered row between the granule and the molecular cortex layers. These neurons are 
characterized by one of the most sophisticated dendritic tree which allows them to integrate numerous signals from the cerebellar and brainstem circuitry.

The Purkinje cells are classified as inhibitory neurons as they release the neurotransmitter GABA $(\gamma$-aminobutyric acid). Their major projections, directed towards the deep cerebellar nuclei, work by inhibiting or reducing the neuronal firing rate particularly of the dentate nucleus $[39,41]$. In addition PCs receive excitatory synapses from several extracerebellar locations, mainly from the inferior olive via climbing fibres that, through the pons and the inferior cerebellar peduncle, enter from the brainstem into the cerebellum [32]. In turn, the inferior olivary nucleus innervates the deep cerebellar nuclei, including the dentate nucleus, thereby completing the Purkinje-Olivo-Dentate network (PODn) [10, 22,35,40].

As regards the ontogenesis, the PCs arise in humans, together with the deep cerebellar nuclei, from the ventricular germinal zone of the metencephalic alar plate at the beginning of the fifth week of development. Only in postmitotic phases, between 9 and 13 gestational weeks, the PCs migrate to their definitive sites. The dorsolateral part of the alar plate, known as "rhombic lip", and precisely the "lower rhombic lip" gives rise to a set of cell migrations that will result in formation of the inferior olivary nucleus in the medulla oblongata [5].

Both the embryological and anatomical links of PCs with the dentate and the inferior olivary nuclei are sustained by the particular absence of expression in the mature neurons of all these structures, of the neuronal nuclear antigen (NeuN). This antigen is in fact intensely expressed in postmitotic neurons of the human nervous system $[27,36,42]$.

A further aim of this study was then to evaluate whether the PODn may somehow be involved as a whole in analogous developmental alterations in SIUDS and SIDS.

The specific study protocol, applied to a wide sample of perinatal deaths ( 65 cases, aged from 25 gestational weeks to 12 months of life, died of both known and unknown causes) included an in-depth morphological and immunohistochemical examination of the PCs and of the dentate nucleus in the cerebellum and of the inferior olivary nucleus in the medulla oblongata.

\section{Material and methods}

The material consisted of 65 brains obtained from autopsy of 30 fresh foetuses, ranging from 25 to 40 gestational weeks, and 35 neonates and infants, died from 2 postnatal days to 12 months, prevalently from the Italian Lombardy region.

The subjects underwent a complete autopsy, including examination of the placental disk, umbilical cord and foetal membranes. All autopsies were performed 1 to 2 days following death, according to the Police Mortuary Regulations in Italy, with no significant differences in post-mortem intervals between subjects.

In all cases, an in-depth histological examination of the cardiorespiratory autonomic nervous system was made, in application of the guidelines applied in our Research Centre of the Milan University [29,30].

In particular, the cerebellum, the primary target of this study, was excised from the pons by dividing the peduncles. Then, sections at intervals of $60 \mu \mathrm{m}$ of both cerebellar hemispheres were obtained. Care was taken to examine corresponding folia from different subjects whenever possible.

Transverse sections at the same intervals were obtained also from the midbrain, pons and medulla oblongata, where the main brainstem structures to be examined, including the inferior olivary nucleus, are located.

All the samples were fixed in $10 \%$ phosphatebuffered formalin, embedded in paraffin and serially sectioned. For each level, six-seven $5 \mu \mathrm{m}$ sections were obtained, two of which were assigned to the histological examination using hematoxylin-eosin and Klüver-Barrera stains; adjacent paraffinized sections were submitted to immunohistochemical procedures for the evaluation of the NeuN, to point out a possible deviation from the normal negative pattern of expression [34], and, when the PC recognition was uncertain, of the Calbindin, a neurochemical hallmark of these cells [20]. The remaining sections were saved and stained as deemed necessary for further investigations.

In 47 cases, death was classified as totally unexplained, after an in-depth microscopic study according to our protocol. A diagnosis of SIUDS was established for 21 foetuses and a diagnosis of SIDS for 26 infants. In the remaining 18 cases ( 9 foetuses and 9 infants, forming the "Control group") a cause of death was 
Table I. Case profiles of the study

\begin{tabular}{lcc}
\hline Subjects & Age range & Death diagnosis \\
\hline $\begin{array}{l}\text { Foetuses } \\
(n=30)\end{array}$ & $25-40 \mathrm{gw}$ & $\begin{array}{c}\text { SIUDS }(n=21) \\
\text { Controls } \\
\end{array}$ \\
& $27-40 \mathrm{gw}$ & $\begin{array}{c}\text { chorioamnionitis }(n=4) \\
\text { congenital heart diseases }(n=5)\end{array}$ \\
\hline $\begin{array}{l}\text { Infants } \\
(n=35)\end{array}$ & $0-12 \mathrm{~m}$ & $\begin{array}{c}\text { SIDS }(n=26) \\
\text { Controls } \\
\text { pneumonia }(n=4)\end{array}$ \\
& $1-11 \mathrm{~m}$ & $\begin{array}{c}\text { congenital heart diseases }(n=4) \\
\text { sepsis }(n=1)\end{array}$ \\
\hline
\end{tabular}

gw - gestational week, $m$ - month

SIUDS - sudden intrauterine unexplained death syndrome, SIDS - sudden infant death syndrome

formulated at autopsy (respiratory infections, cardiomyopathies, sepsis in infant deaths; cardiomyopathies and chorioamnionitis in foetal deaths). Table I summarizes the case profile of this study.

Complete clinical histories in all the cases were collected. Additionally, mothers completed a questionnaire on their smoking habits, detailing the number of cigarettes smoked before, during and after pregnancy. Twenty-two out of the 47 SIUDS/SIDS mothers (47\%) were active smokers before and during the pregnancy, smoking $>3$ cigarettes/day. The remaining 25 mothers (53\%) reported no history of cigarette smoking. Four mothers of the Control group $(22 \%)$ reported a smoking habit, while 14 mothers (78\%) were non-smokers.

Consent. Parents of all the victims of the study provided written informed consent to autopsy, with the Milan University L.Rossi Research Centre institutional review board approval.

\section{Immunohistochemical neuronal nuclear antigen detection}

Selected sections from paraffin-embedded tissue blocks were stained using commercially supplied mouse monoclonal antibody against the neuronal nuclear antigen NeuN (Millipore Chemicon International, MAB377). A standard avidin-biotin complex technique was used with peroxidase-diaminobenzidine to visualize and develop the antigen-antibody reaction. The antibody dilution at 1 : 100 was used. Incubating solutions were boiled in citrate buffer $\mathrm{pH}$ 6.0, in a microwave oven, for $5 \mathrm{~min}$ at high power, then $5 \mathrm{~min}$ at $50 \%$ power, and finally cooled for 20 min. Sections were counterstained lightly with Mayer's hematoxylin.

\section{Immunohistochemical calbindin detection}

A mouse IgG1 monoclonal antibody against calbindin-D28k (clone CL-300, Sigma Immunochemicals, St. Louis, MO), a specific PC marker in the cerebellum, was used in selected paraffin sections, at dilution of $1: 200$. As the secondary antibody, anti-rabbit IgG was used. For visualization of the reaction sites, immunohistochemical staining was carried out by the peroxidase linked avidin-biotin complex (ABC) method. Blocking of endogenous peroxidase activity was obtained with $0.08 \%$ hydrogen peroxidase $\left(\mathrm{H}_{2} \mathrm{O}_{2}\right)$ in methanol for 5 minutes. In order to block unspecific binding, incubation with $(1: 10)$ normal goat serum in $0.1 \mathrm{M} \mathrm{PBS}$, $\mathrm{pH} 7.2$ was performed.

\section{Microscopic evaluations}

The histological examination, besides the evaluation of the developmental steps of the PC layer, included the classification of the PCs according to their cytological features. They were categorized in "normal cells" with a distinct stained nucleus, and "damaged cells" with swollen, necrotic, shrunken or dark degeneration (classification adapted from Hausmann et al. [19]). The morphology of 150-200 cells per case along randomly selected segments of the PC layer was examined at light microscope in Klüver-Barrera stained histological sections, at $\times 20$ magnification, and at $\times 100$ magnification when more in-depth cytological information was needed.

As regards the immunohistochemical analysis, only the cells with intense brown NeuN-immunostaining were considered to be really positive. 
Histological and immunohistochemical observations were carried out by two independent blinded pathologists. Comparison among the results was performed employing Kappa statistics (Kappa Index - KI) to evaluate the inter-observer reproducibility. The Landis and Koch system [21] for $\mathrm{K}$ interpretation was used, where 0 to 0.2 is slight agreement, 0.21 to 0.40 indicates fair agreement, 0.41 to 0.60 is moderate agreement, 0.61 to 0.80 is strong or substantial agreement, and 0.81 to 1.00 indicates very strong or almost perfect agreement (a value of 1.0 being perfect agreement). The application of this method revealed a very satisfactory Kappa Index $(\mathrm{KI}=0.85)$.

\section{Statistical analysis}

The statistical significance of direct comparison between groups was determined using analysis of variance (ANOVA). Statistical calculations were carried out with an SPSS statistical software (version 11.0; SPSS Inc., Chicago, IL, USA). The selected threshold level for statistical significance was $P<0.05$.

\section{Results}

According to the purpose of this study, below we indicate only in-depth morphological and immunohistochemical observations related to the PCs. Developmental steps of all the cerebellar cortex layers can be found in our previous works [24-26].

\section{Chronological changes in Purkinje cells during the human cerebellar cortex development}

First we highlighted progressive developmental stages of the PCs maturation in control cases from the foetus to the infant.

At the earliest observation, around the $25^{\text {th }}$ gestational week, the PCs result in a pluristratified, poorly defined row between sketchy molecular and internal granular layers (Fig. 1A).

Successively, at 28-30 weeks, the PCs form an ordered 3-4 cells thick layer of round immature larger neurons with several short processes (Fig. 1B). Around the $35^{\text {th }}$ week of gestation, a single layer of round or pear-shaped PCs, increased in size, is recognized. The PCs have prominent primary dendrites arranged in a parallel pattern in the molecular layer (Fig. 1C).
The PC layer at 38-40 weeks shows numerous large cells, polygonal in shape and with a wide branched arborization. Frequently it is possible to distinguish transversal branches in the molecular layer with a single primary process that extends towards the pial surface (Fig. 1D). Shrunken neurons without evident nucleus were sometimes present. This cytoarchitecture is maintained at birth and in the first year of life.

PCs display negative NeuN-immunoreactivity at all ages, in spite of the external and internal granular layers of the cerebellar cortex that are strongly positive (Fig. 2).

\section{Purkinje cells alterations in sudden intrauterine unexplained death syndrome and sudden infant death syndrome}

Thirteen victims of sudden death showed the above delineated cytoarchitectural and biological features corresponding to developmental age. Nevertheless, abnormal patterns of PC maturation were identified in 34 cases (72\%).

Precisely, in 12 subjects diagnosed as SIDS, aged 3-6 months, and in 8 foetuses suddenly died between 36 and 40 gestational weeks, heterotopic scattered PCs, isolated or in clusters, were visible within the molecular and/or granular layers and even positioned over the external granular layer. These misplaced cells, identified as PCs by their immunoreactivity for Calbindin-D28k, showed an immature oval structure with substantial reduction of the dendritic tree (Fig. 3).

A loss of PCs for extensive sections of the cerebellar cortex (hypoplasia) was observed in 11 cases (5 SIUDS and 6 SIDS) (Fig. 4A). Conversely, in 6 further SIDS cases (aged 3-4 months) and in 3 late SIUDS cases (38-40 gestational weeks) the PC layer was disorganized with densely packed roundish cells in a pluristratified structure (hyperplasia of the PCs) (Fig. 4B). Mitotic figures in adjacent tracts were not uncommon findings, prevalently in SIDS cases (Fig. 5). Nevertheless, more frequently (precisely in 12 SIUDS and 14 SIDS) the PCS were classified as "damaged cells", as they did not exhibit the nucleus and showed shrunken morphology with intensive dark staining of the cytoplasm lacking arborization (Fig. 6).

Only in 3 control cases, occasional PC alterations were observed. On the whole, a significant decreased 
A
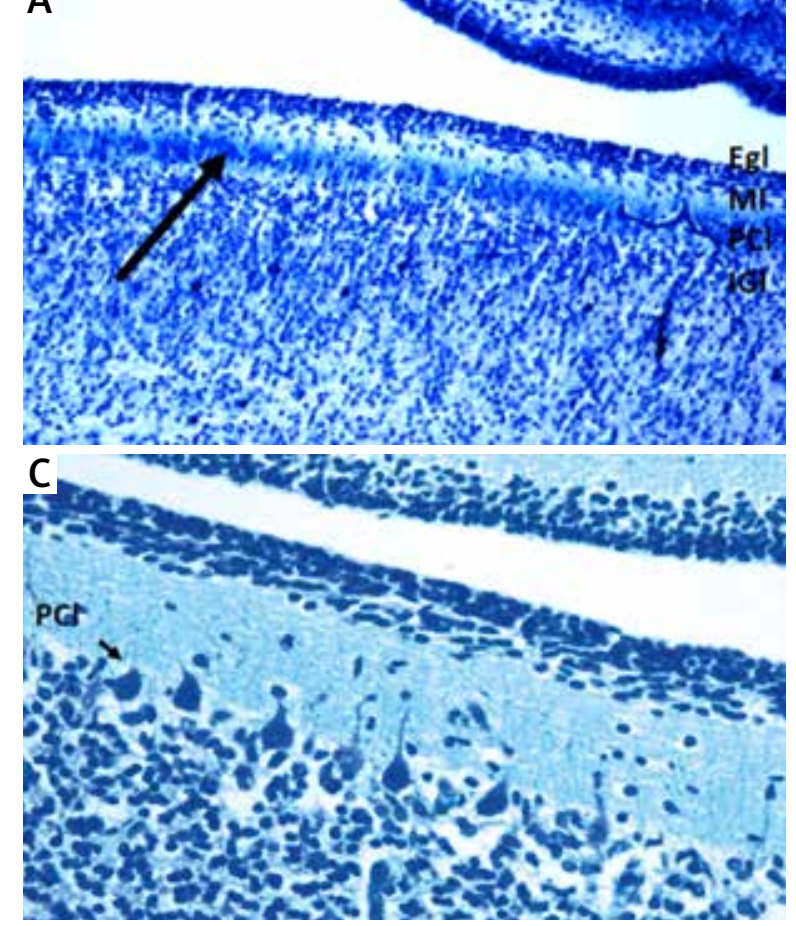
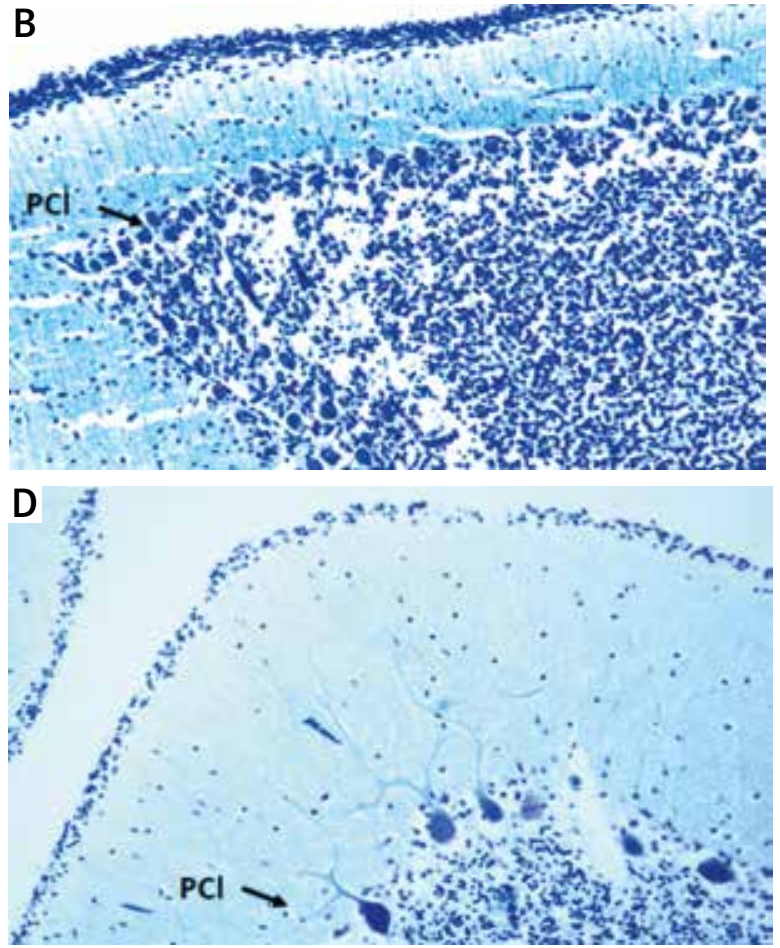

Fig. 1. Developmental steps of the PCs. A) Cerebellar cortex of a 25-gestational-week foetus of the control group. The arrow indicates a poorly defined pluristratified PC layer between the molecular layer and the internal granular layer. B) Round PCs arranged in a 3-4 row layer in the cerebellar cortex of a 30 gestational-week-old foetus. C) Regular monostratified arrangement of PCs with primary dendrites running towards the external granular layer in a foetus died at 34 gestational weeks. D) PCs in a newborn died in the first days of life, showing wide arborization of the processes. Klüver-Barrera stain. EGI - external granular layer, IGI - internal granular layer, MI - molecular layer, PCl - Purkinje Cell layer. Magnification: A), B) 10x; C), D) $20 \times$.

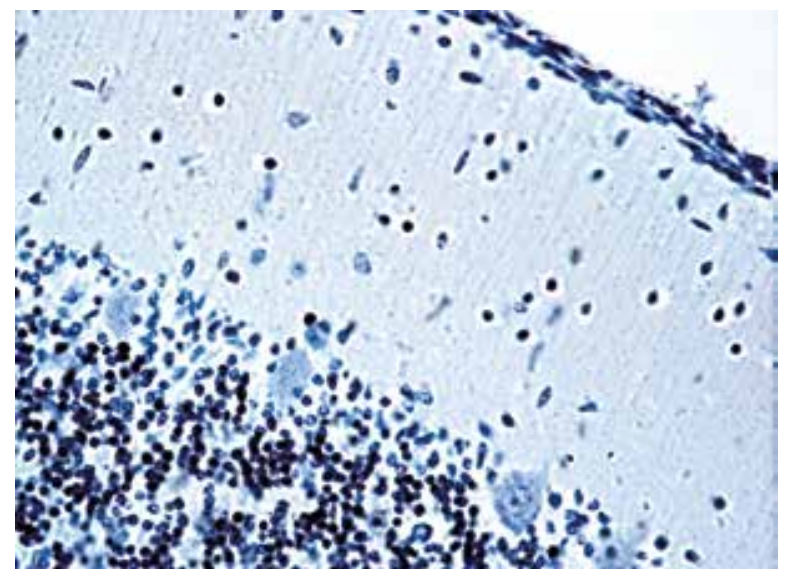

Fig. 2. NeuN-immunonegative PCs in a 2-monthold infant. Conversely note the intense immunopositivity of both the internal and external granular layer neurons. NeuN - immunostaining; magnification: 20x. incidence of intact PCs was detected in the cerebellar cortex of suddenly dead victims compared to controls $(p<0.01)$.

Finally, positive NeuN-immunolabeling resulted in morphologically immature PCS of 16 SIDS (range of age: from 3 to 8 months) and 10 SIUDS cases (3439 gws) (Fig. 7). The staining was mainly present in the nucleus, but also the cytoplasm was immunoreactive, though to a lesser extent.

Frequently the above-reported alterations were combined in the same case. Noteworthy was a significant association highlighted between PC alterations and cigarette smoke exposure $(p<0.01)$. In fact, in 20 out of the 22 SIUDS/SIDS cases with a smoking mother, defective development of the PC layer was present. The association between the presence of damaged PCs and smoke absorption was very significant $(p<0.01)$. Table II summarizes all the findings. 

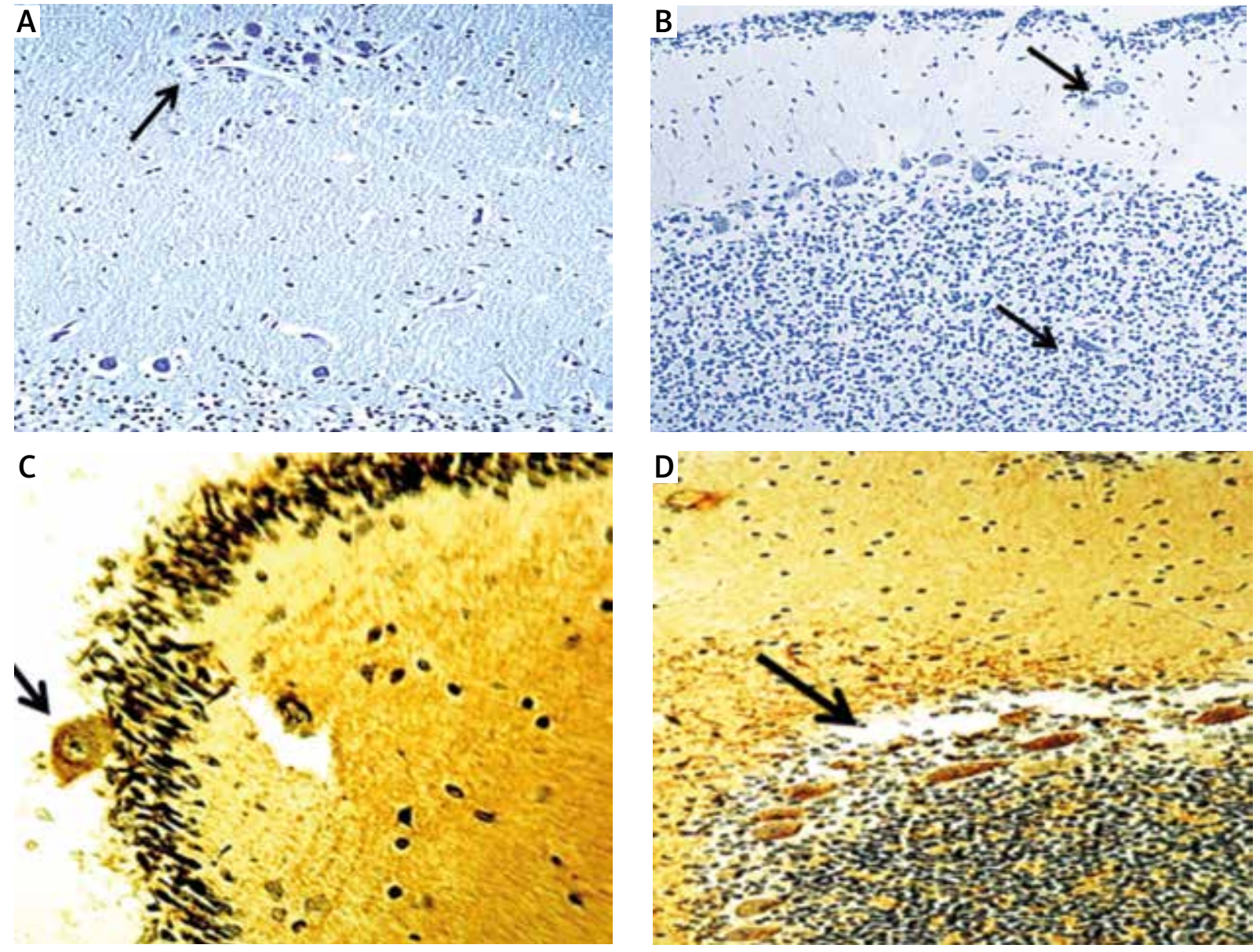

Fig. 3. Heterotopic PCs. Arrows indicate: in A) a cluster of PCs in the molecular layer (3-month-old SIDS case); in B) isolated PCs in the molecular layer and intermingled with the internal granular layer neurons (39-gestational-week foetus); in C) a misplaced neuron overlooking the external granular layer identified as PC by its immunoreactivity for calbindin (37-gestational-week foetus); in D) normal layer of PCs calbindin-immunopositive as control. A) and B) Klüver-Barrera stain; C) and D) calbindin-D28k immunohistochemistry; magnification: A), B) and D) 20x; C) 40x.

\section{Additional results on cerebellum and brainstem}

The present PC analyses were complemented by findings in the structurally and ontogenetically linked structures in the cerebellum and brainstem. Interesting was the observation of hypodevelopment of both the dentate nucleus and the inferior olivary nucleus with neuronal degeneration, in 8 out of the 11 cases with PC hypoplasia (Fig. 8).

The routine histological examination of other brainstem structures led to the diagnosis in several cases, and above all in SIUDS, of hypoplasia/agenesis of the arcuate, the pre-Bötzinger, the inferior olivary, the Kölliker-Fuse, the parafacial and the serotonergic raphé nuclei, but without any specific correlation with the PODn alterations.

\section{Discussion}

During the last decade our research Centre reported developmental abnormalities of different nuclei and/or structures in the brainstem and cerebellum of SIUDS and SIDS victims. In particular, a group of our studies was performed to highlight alterations of the cerebellar cortex, since its neat layered structure and its developmental pattern over a long period of time, extending from the early embryonic life until the first postnatal year, make this structure a favourite field of research [24-26]. 

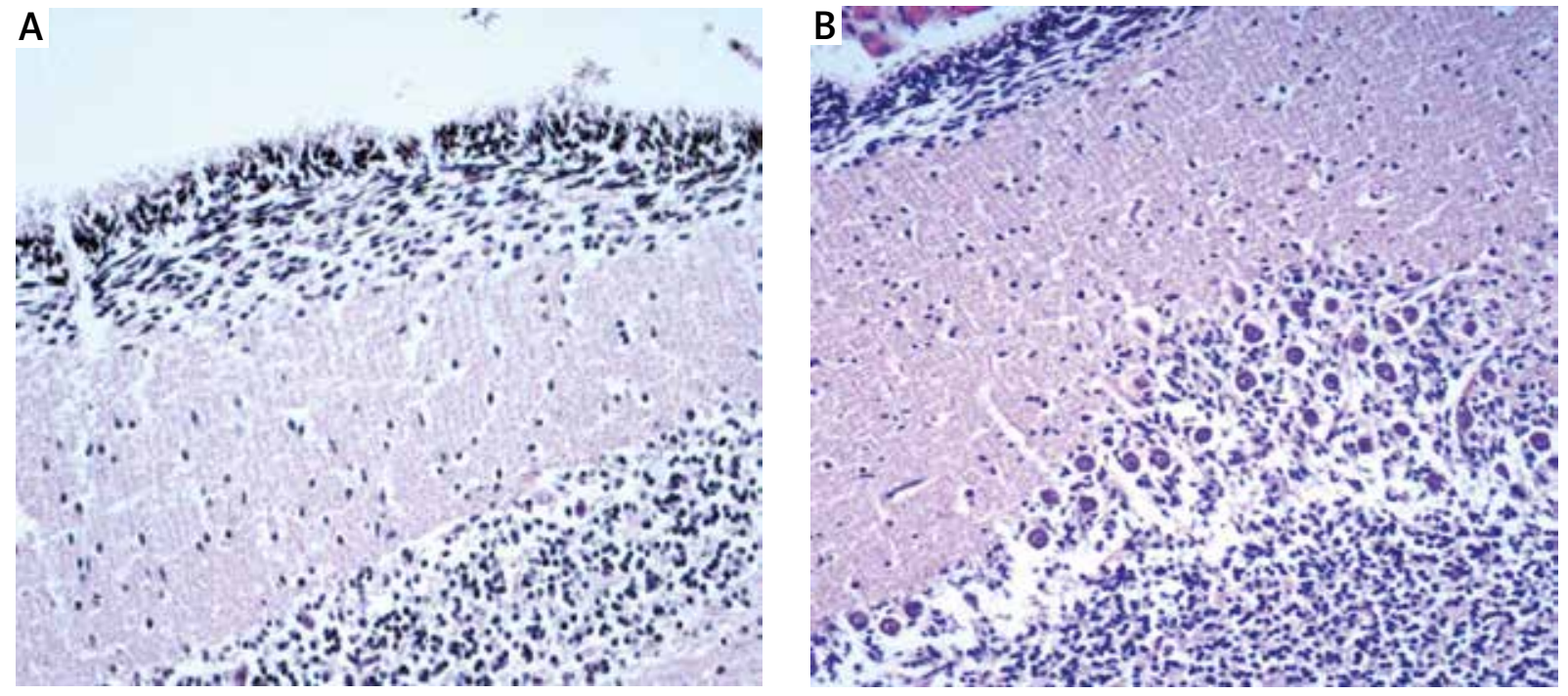

Fig. 4. PC numerical alterations. A) PC depletion in a late SIUDS case of 39 gestational weeks (hypoplasia). B) Pluristratified disposition of roundish PCs in a SIDS victim died at 2 months (hyperplasia). Klüver-Barrera stain; magnification: A) and B) 20x.


In the present study we expanded the information hitherto obtained on the PCs adding specific developmental alterations, previously not well outlined, in sudden unexplained perinatal deaths. Precisely, we observed patterns of heterotopia, hypoplasia, hyper-

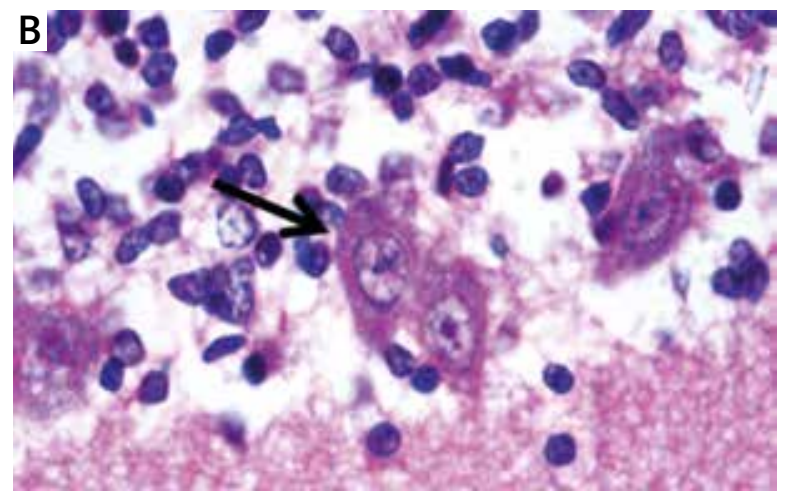

Fig. 5. PCs in mitotic phases (telophases). In A) and B) mitotic figures observed in 2 SIDS died at 3 months; in C) a telophase in a SIUDS of 38 gestational weeks (see arrows). A) and C) Klüver-Barrera stain; B) hematoxylin-eosin stain; magnification: 100x.

plasia, presence of mitotic figures and/or shrunken features, in addition to anomalous positive NeuN immunoexpression in PCs. Many of these defects could be a result of disruption of radial neuronal migration systems occurring in early human embry- 

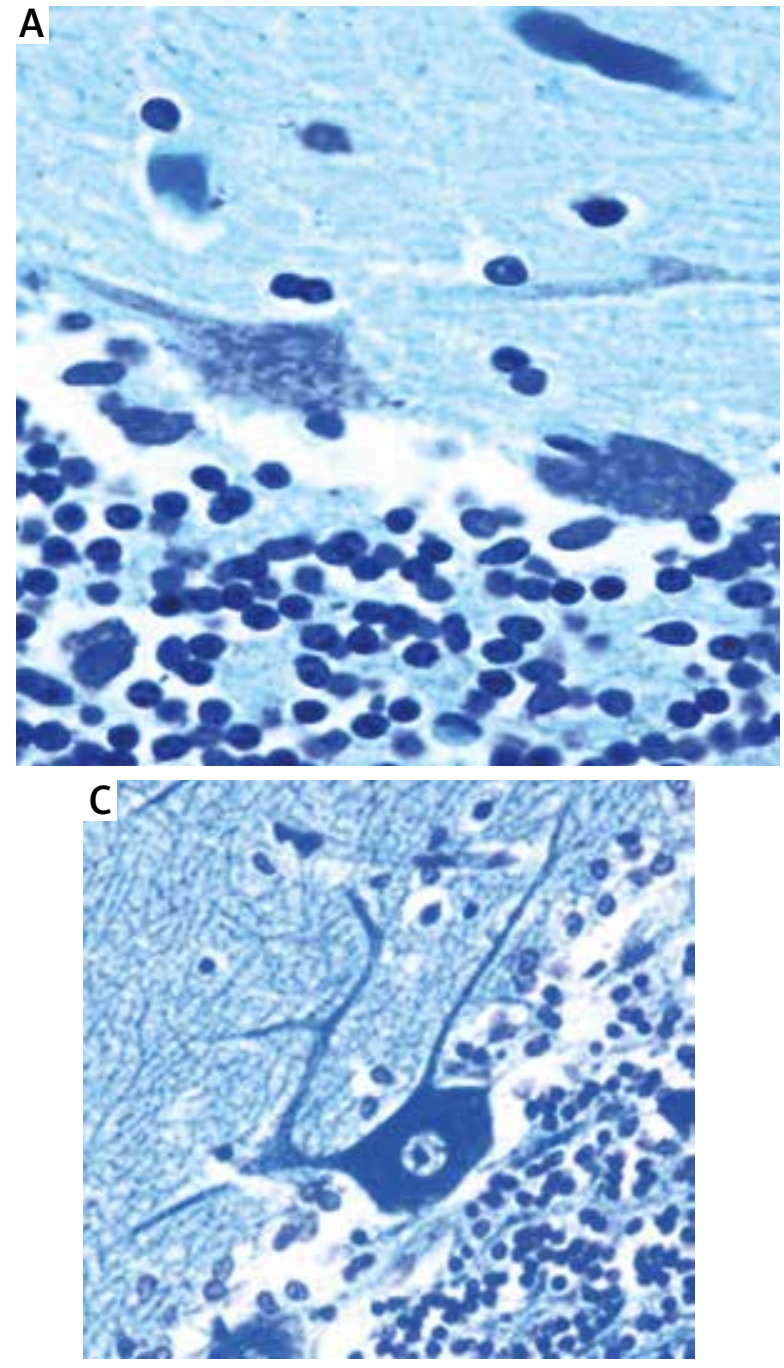

onic stages when the PCs migrate from the germinal ventricular zone of the metencephalic alar plates, where they undergo terminal mitoses, into the cerebellar wall $[18,37,43]$.

The formation of the PC layer is indeed a complex process that requires interactions between multiple neuronal populations and precise migratory patterns established under a definite glia guidance. The presence of PCs in heterotopic sites, their decreased or increased number and the persistence of mitotic features can be a result of an arrested or premature and erroneous neuronal migration in the foetal cerebellum [9]. A similar interpretation was proposed by Laure-Kamionowska and Maślińska [23] in a large study on morphologic features of the cerebellum in perinatal life. These authors considered in fact the frequent finding of $P$ neurons irregularly dispersed in the molecular and granular layers as

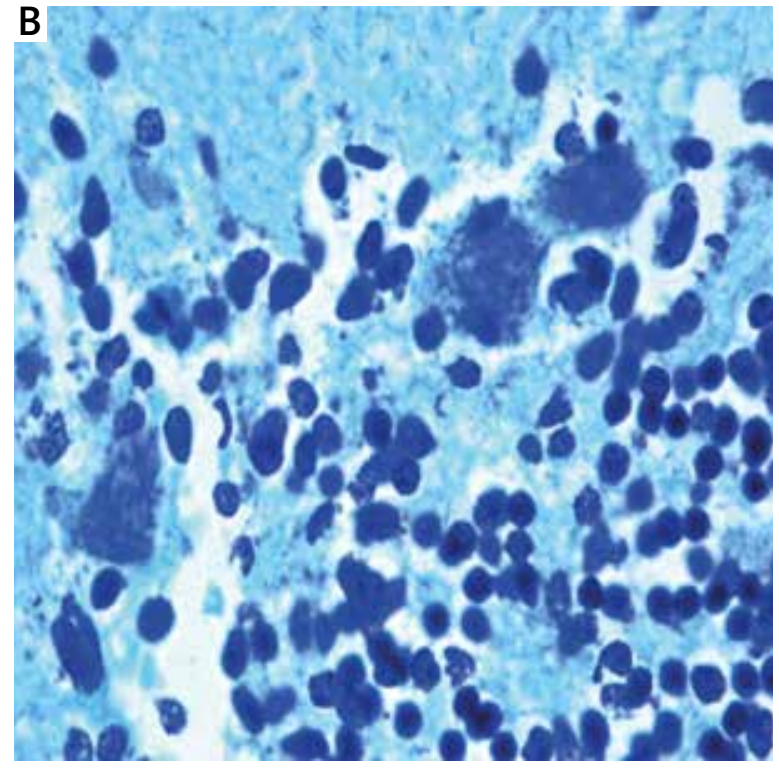

Fig. 6. A) and B) Irregular shrunken morphology of PCs without a distinct nucleus in 2 SIDS victims (both 5 month old). In C) normal features of a PC with well-evident nucleus and nucleolus from a control age-matched case. Klüver-Barrera stain; magnification: 100x.

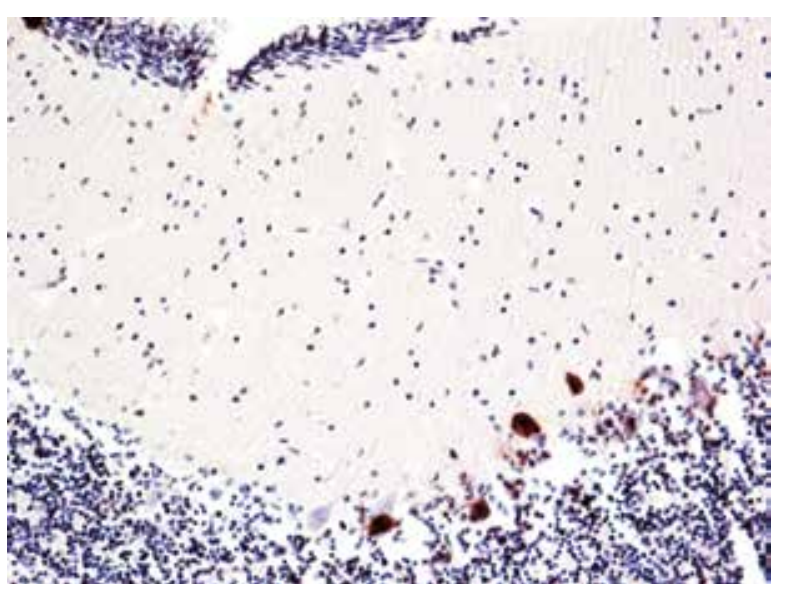

Fig. 7. NeuN-immunopositive PCs in a 3-monthold SIDS case. The staining is present in both nuclei and cytoplasm of the cells. NeuN immunostaining; magnification: 40x. 

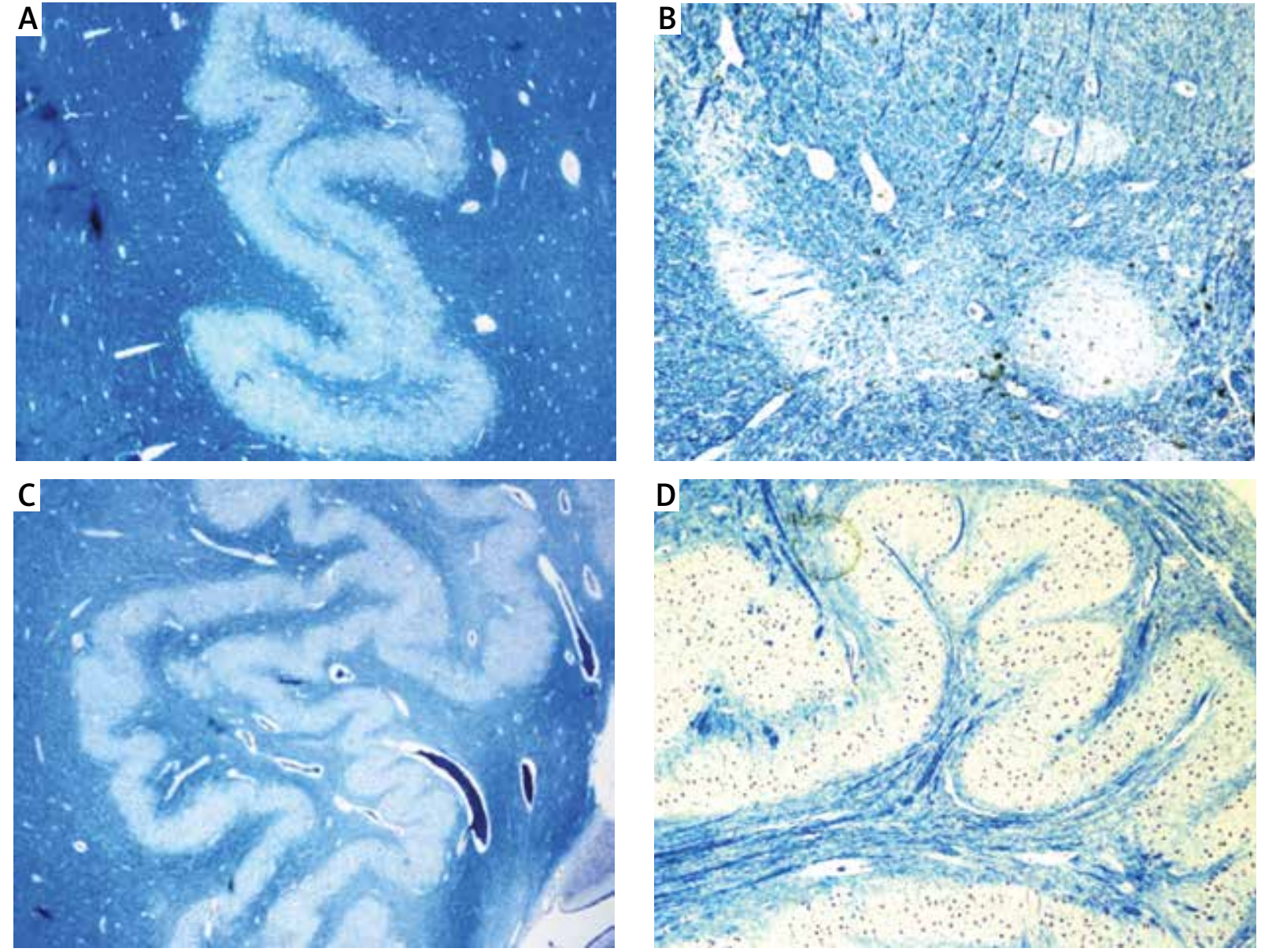

Fig. 8. A) and B) Hypoplasia of the cerebellar dentate nucleus and of the medullary inferior olivary nucleus, respectively, in a SIDS case of 4 months (also affected by PC layer hypoplasia). C) and D) normal structure of the dentate nucleus and of the inferior olivary nucleus, respectively, in an age-matched infant control. Klüver-Barrera stain; magnification: 10x.

Table II. Morphological and immunohistochemical alterations of the PCs in SIUDS, SIDS and Control cases

\begin{tabular}{lccc}
\hline Alterations of the PCS & SIUDS & SIDS & Controls \\
$n=21$ & $n=26$ & $n=18$ \\
Total number of cases, $n=34^{*}$ & $8(4)$ & $12(5)$ & $2(0)$ \\
\hline Heterotopia & $5(2)$ & $6(3)$ & $1(1)$ \\
\hline Decreased number (hypoplasia) & $3(2)$ & $6(2)$ & $0(0)$ \\
\hline Increased number (hyperplasia) & $2(1)$ & $5(3)$ & $0(0)$ \\
\hline Mitosis & $12(9)$ & $14(11)$ & $3(1)$ \\
\hline $\begin{array}{l}\text { Damaged cytological features } \\
\text { (shrunken aspect, indistinct nucleus, darkened degeneration) }\end{array}$ & $10(5)$ & $16(6)$ & $0(0)$ \\
\hline Positive NeuN expression & & & \\
\hline
\end{tabular}

When comparing SIUDS/SIDS groups vs. Controls, $p<0.01$

*Individual victims may display combination of the PC alterations

NeuN - neuronal nuclear antigen, PC - Purkinje cell, SIUDS - sudden intrauterine unexplained death syndrome, SIDS - sudden infant death syndrome

A total of 34 out of the 47 SIUDS/SIDS cases had one or more developmental defects of the cerebellar PC layer. Twenty of the 22 cases of SIUD/SIDS with a smoking mother belong to this group of 34 cases. In brackets the number of cases with a smoking mother is indicated, for every type of alteration (always considering that individual victims may display any combination of the CP alterations). 
a result of impaired migration of neuronal precursors in improper places. The NeuN intense immunoreactivity can be instead interpreted as a deep disorder of the normal developmental programs of these cells. The PCs in fact cannot usually be recognized by the NeuN antibody in the postmitotic phases, unlike other neurons of the human nervous system which migrate from the birthplace to their final position $[27,36,42]$.

Our findings could be a consequence of exposure to toxic agents. It is known in fact that the protracted development of the cerebellar cortex makes this structure particularly vulnerable to a broad spectrum of extrinsic injuries, such as cigarette smoke, air pollution, ionizing radiations, drugs, alcohol, etc. $[11,12,28]$. In particular, the period over the $28^{\text {th }}$ week of gestation in humans seems to be of crucial importance for the maturation of PCs and for the development of their structural and functional connections. Particularly from this age the PCs may be affected by acquired disorders [13]. Accordingly, we have observed PC alterations in subjects older than 28 gestational weeks with a high frequency of smoking mothers, prevalently died in Lombardy, a highly polluted Italian region.

Although the cerebellar cortex activity, likely ranging from the control and coordination of movements to autonomic functions, is still poorly defined, an inhibitory function to respiration, especially during hypercapnia, has been recognized to the PCs. Xu et al. [45] in experimental studies on rats demonstrated in fact that degeneration of PCs enhances eupneic and hypercapnic ventilation. Morphological alterations of the PCs have been reported also as a consequence of hypoxic insults $[2,4,19]$. In particular, shrunken, swollen, necrotic PCs with lack of a distinct nuclear staining and/or dark cell degeneration are considered primary manifestations of chronic hypoxic damage [19]. Thus, the PC alterations here observed in high percentages of SIUDS and SIDS could be determined by chronic hypoxic exposure.

The major risk factor for chronic hypoxia in perinatal periods is the exposure to maternal cigarette smoke. Nicotine is one of the few lipid-soluble substances among many cigarette toxic compounds that can pass through the blood-brain barrier and act directly on the foetal brain [16]. Thus, nicotine is capable of producing a wide spectrum of abnormalities of neuronal cells, particularly of the developing PCs $[7,8]$. One major action of nicotine is the interaction with nicotinic receptors, a subpopulation of the acetylcholine receptors (nAChRs), and in particular with the $\alpha 4$ and $\alpha 7$ subunits that are strongly expressed in PCs [15].

In agreement with these considerations, the results of our study showed a significant correlation between prenatal cigarette smoke absorption and developmental alterations of the PC layer. The specific study of the nicotinic receptors in our series is now in progress in our laboratory and it will be the subject of a forthcoming publication.

The vulnerability of PCs seems to have an impact also on the dentate and the inferior olivary nuclei, given their mutual connections and the common origin from the same embryological compartment of the neural tube. We observed in fact in $34 \%$ of the SIUDS/SIDS cases, developmental alterations of all these structures. Furthermore, likewise to PCs, also the inferior olivary nucleus and the dentate nucleus may play a critical role in modulating the respiratory pattern $[17,33,38,44]$.

In conclusion, this work suggests an important role of the PODn in the physiology of breathing. While it may be plausible that the cerebellar-medullary defects reported here give rise to respiratory inhibition after birth, and consequently to sudden neonatal and infant death, we can reasonably speculate whether ventilatory alterations during intrauterine life can lead to foetal death. We know that an immature respiratory-related neuronal network is active before birth and controls breathing-like rhythmic movements, necessary to favour the lung development $[3,6]$. However, intrauterine respiratory alterations would not be sufficient to justify a foetal loss. One possibility that we strongly support is that the PCs together with the connected structures participate not only in respiratory modulation but, more extensively, in the general control of all the vital functions. Consequently, disruption of the ascending and descending pathways between cerebellum and medulla, likely imputable to smoke exposure in utero, can produce severe dysregulation in the autonomic nervous system, triggering a sudden death mechanism in vulnerable periods of pre- and postnatal life.

\section{Acknowledgements}

This study was supported by the Italian Health Ministry in accordance with Law 31/2006 "Regula- 
tions for Diagnostic Post Mortem Investigation in Victims of Sudden Infant Death Syndrome (SIDS) and Unexpected Foetal Death" and by the Convention between the Provincia autonoma di Trento and the "Lino Rossi" Research Centre of the Milan University, Italy. The authors thank Dr. Graziella Alfonsi for the excellent technical cooperation.

\section{Disclosure}

\section{Authors report no conflict of interest.}

\section{References}

1. Armstrong CL, Hawkes R. Pattern formation in the cerebellar cortex. Biochem Cell Biol 2000; 78: 551-562.

2. Barenberg P, Strahlendford $\mathrm{H}$, Strahlendford J. Hypoxia induces an excitatory-type of dark cell degeneration in cerebellar Purkinje neurons. Neurosci 2001; 40: 245-254.

3. Barness EG. Respiratory system. In: Potter's Pathology of the Fetus and Infant. Barness EG (ed.). Mosby, St. Louis 1997, pp. 712-773.

4. Bartschat S, Fieguth A, Könemann J, Schmidt A, Bode-Jänisch S Indicators for acute hypoxia - an immunohistochemical investigation in cerebellar Purkinje-cells. Forensic Sci Int 2012; 223: 165-170.

5. Bayer SA, Altman J, Russo RJ, Zhang X. Embryology. In: Duckett S (ed.). Pediateric Neuropathoogy. Williams \& Wilkins, Baltimore 1995, pp. 54-107.

6. Boddy K, Dawes GS. Fetal breathing. Br Med Bull 1975; 31: 3-7.

7. Chen WJ, Edwards RB, Romero RD, Parnell SE, Monk RJ. Longterm nicotine exposure reduces Purkinje cell number in the adult rat cerebellar vermis. Neurotoxicol Teratol 2003; 25: 329334.

8. Chen WJ, Parnell SE, West JR. Neonatal alcohol and nicotine exposure limits brain growth and depletes cerebellar Purkinje cells. Alcohol 1998; 15: 33-41.

9. Chevassus-au-Louis N, Represa A. The right neuron at the wrong place: biology of heterotopic neurons in cortical neuronal migration disorders, with special reference to associated pathologies. Cell Mol Life Sci 1999; 55: 1206-1215.

10. Courville J. Distribution of olivocerebellar fibers demonstrated by a radioautoradiographic tracing method. Brain Res 1975; 95 : 253-263.

11. ten Donkelaar HJ, Lammens M, Wesseling P, Thijssen HO, Renier WO. Development and developmental disorders of the human cerebellum. J Neurol 2003; 9: 1025-1036.

12. Fonnum F, Lock EA. Cerebellum as a target for toxic substances. Toxicol Lett 2000; 112-113: 9-16.

13. Friede RL. Developmental Neuropathology. 2nd ed. Springer Verlag, Berlin 1989, p. 363.

14. Gadson DR, Emery JL. Quantitative morphological studies of developing human cerebellar cortex in various disease states. Arch Dis Child 1976; 51: 964-967.

15. Graham A, Court JA, Martin-Ruiz CM, Jaros E, Perry R, Volsen SG, Bose S, Evans N, Ince P, Kuryatov A, Lindstrom J, Gotti C, Perry EK.
Immunohistochemical localisation of nicotinic acetylcholine receptor subunits in human cerebellum. Neuroscience 2002; 113: 493-507.

16. Gressens P, Laudenbach V, Marret S. Mechanisms of action of tobacco smoke on the developing brain. J Gynecol Obstet Biol Reprod (Paris) 2003; 32 (1 Suppl): 30-32.

17. Gruart A, Delgado-García JM. Respiration-related neurons recorded in the deep cerebellar nuclei of the alert cat. Neuroreport 1993; 3: 365-368.

18. Hatten ME. Riding the glial monorail: a common mechanism for glial-guided neuronal migration in different regions of the developing mammalian brain. Trends Neurosci 1990; 13: 179 184.

19. Hausmann R, Seidl S, Betz P. Hypoxic changes in Purkinje cells of the human cerebellum. Int I Legal Med 2007; 121: 175-183.

20. Kim BJ, Lee SY, Kim HW, Park EJ, Kim J, Kim SJ, So I, Jeon JH. Optimized immunohistochemical analysis of cerebellar Purkinje cells using a specific biomarker, calbindin d28k. Korean J Physiol Pharmacol 2009; 13: 373-378.

21. Landis RJ, Koch GG. The measurement of observer agreement for categorical data. Biometrics 1977; 33: 159-174.

22. Lapresle J, Hamida MB. The dentato-olivary pathway. Somatotopic relationship between the dentate nucleus and the contralateral inferior olive. Arch Neurol 1970; 22: 135-143.

23. Laure-Kamionowska M, Maślińska D. Cerebellar cortical neurons misplaced in the white matter due to disturbed migration during development of human brain. Folia Neuropathol 2011; 49: 282-294.

24. Lavezzi AM, Ottaviani G, Matturri L. Ontogenesis of human cerebellar cortex and biopathological characterization in sudden unexplained fetal and infant death. Virchows Arch 2007; 450: 31-40.

25. Lavezzi AM, Ottaviani G, Mauri M, Matturri L. Alterations of biological features of the cerebellum in sudden perinatal and infant death. Curr Mol Med 2006; 6: 429-435.

26. Lavezzi AM, Ottaviani G, Terni L, Matturri L. Histological and biological developmental characterization of the human cerebellar cortex. Int I Dev Neurosci 2006; 24: 365-371.

27. Lavezzi AM, Corna MF, Matturri L. Neuronal nuclear antigen (NeuN): a useful marker of neuronal immaturity in sudden unexplained perinatal death. J Neurol Sci 2013; 329: 45-50.

28. Lewandowska E, Stępień T, Wierzba-Bobrowicz T, Felczak P, Szpak GM, Pasennik E. Alcohol-induced changes in the developing cerebellum. Ultrastructural and quantitative analysis of neurons in the cerebellar cortex. Folia Neuropathol 2012; 50: 397-406.

29. Matturri L, Ottaviani G, Lavezzi AM. Techniques and criteria in pathologic and forensic-medical diagnostics of sudden unexpected infant and perinatal death. Am J Clin Pathol 2005; 124 : 259-268.

30. Matturri L, Ottaviani G, Lavezzi AM. Guidelines for neuropathologic diagnostics of perinatal unexpected loss and sudden infant death syndrome (SIDS). A technical protocol. Virchows Arch 2008; 452: 19-25.

31. McKay BE, Engbers JD, Mehaffey WH, Gordon GR, Molineux ML, Bains JS, Turner RW. Climbing fiber discharge regulates cerebel- 
lar functions by controlling the intrinsic characteristics of Purkinje cell output. J Neurophysiol 2007; 97: 2590-2604.

32. McKay BE, Turner RW. Physiological and morphological development of the rat cerebellar Purkinje cell. J Physiol 2005; 567: 829-850.

33. Nogami M, Takatsu A, Endo N, Ishiyama I. Immunohistochemical localization of c-fos in the nuclei of the medulla oblongata in relation to asphyxia. Int J Legal Med 1999; 112: 351-354.

34. Rakic P, Sidman RL. Histogenesis of cortical layers in human cerebellum, particularly the lamina dissecans. J Comp Neurol 1970; 139: 473-500.

35. Ruigrok TJH, Voogd J. Organization of projections from the inferior olive to the cerebellar nuclei in the rat. J Comp Neurol 2000; 426: 209-228.

36. Sarnat HB, Nochlin D, Born DE. Neuronal nuclear antigen (NeuN): a marker of neuronal maturation in early human fetal nervous system. Brain Dev 1998; 20: 88-94.

37. Sotelo C, Rossi F. Purkinje cell migration and differentiation. In: Handbook of Cerebellum and Cerebellar Disorders. Manto M, Gruol D, Schmamann J, Koibuchi N, Rossi F (eds.). Springer, New York 2011.

38. Takashima S. Olivocerebellar lesions in infants born prematurely. Brain Dev 1982; 4: 361-366.

39. Teune TM, van der Burg J, de Zeeuw Cl, Voogd J, Ruigrok TJ. Single Purkinje cell can innervate multiple classes of projection neurons in the cerebellar nuclei of the rat: a light microscopic and ultrastructural triple-tracer study in the rat. J Comp Neurol 1998; 392: 164-178.

40. Tolbert DL, Massopust LC, Murphy MG, Young PA. The anatomical organization of the cerebello-olivary projection in the cat. J Comp Neurol 1976; 170: 525-544.

41. Uusisaari M, Knöpfel T. GABAergic synaptic communication in the GABAergic and non-GABAergic cells in the deep cerebellar nuclei. Neuroscience 2008; 156: 537-549.

42. Weyer A, Schilling K. Development and cell type-specific expression of the neuronal marker NeuN in the murine cerebellum. J Neurosci Res 2003; 73: 400-409.

43. Wingate RJT. The rhombic lip and early cerebellar development. Curr Opin Neurobiol 2001; 11: 82-88.

44. Xu F, Frazier DT. Modulation of respiratory motor output by cerebellar deep nuclei in the rat. J Appl Physiol 2000; 89: 996-1004.

45. Xu F, Zhou T, Frazier DT. Purkinje cell degeneration elevates eupneic and hypercapnic ventilation in rats. Cerebellum 2004; 3: $133-140$.

46. Zecevic N, Rakic P. Differentiation of Purkinje cells and their relationship to other components of developing cerebellar cortex in man. J Com Neurol 1976; 167: 27-47. 